The Astrophysical Journal, 654: L135-L138, 2007 January 10

(C) 2007. The American Astronomical Society. All rights reserved. Printed in U.S.A.

\title{
THE POPULATION OF RADIO SOURCES IN THE FIELD OF THE UNIDENTIFIED GAMMA-RAY SOURCE TeV J2032+4130
}

\author{
Josep M. Paredes, ${ }^{1}{ }^{\text {Josep Martí, }}{ }^{2}$ C. H. Ishwara Chandra, ${ }^{3}$ and Valentí Bosch-Ramon ${ }^{4}$ \\ Received 2006 October 20; accepted 2006 November 28; published 2006 December 28
}

\begin{abstract}
$\mathrm{TeV} \mathrm{J} 2032+4130$ is the first extended very high energy gamma-ray source and has remained enigmatic since its discovery because of the lack of identification. We report here deep radio observations covering the TeV $\mathrm{J} 2032+4130$ field and revealing for the first time an extended and diffuse radio emission, as well as a remarkable population of compact radio sources. Some of these radio sources are in positional coincidence with X-ray and optical/IR sources. Future follow-up studies of these new radio sources will likely contribute to solving the mystery of this extended unidentified TeV source.
\end{abstract}

Subject headings: gamma rays: observations — radio continuum: ISM — radio continuum: stars — X-rays: stars

\section{INTRODUCTION}

The new generation of Cerenkov telescopes has revealed the existence of a population of very high energy gamma-ray sources in the Milky Way (Aharonian et al. 2005a, 2006). Some of these sources are well identified and are compact sources like LS 5039 (Aharonian et al. 2005b) or LSI+61 303 (Albert et al. 2006), whereas others still remain without known counterpart. Among the latter there are some extended TeV sources (Aharonian et al. 2002, 2005c), for which all attempts to find their counterparts at other wavelengths have failed, and they are being considered a new population of Galactic sources. Because of the lack of detection of a low-energy counterpart, it has been proposed that hadronic processes instead of leptonic ones could be behind the TeV emission (e.g., Torres et al. 2004).

The so far unidentified source TeV J2032+4130, discovered with the stereoscopic High Energy Gamma Ray Astronomy (HEGRA) array of imaging Cerenkov telescopes in the direction of the Cygnus OB2 star association (Aharonian et al. 2002), is perhaps the most representative within the group of $\mathrm{TeV}$ sources with unknown counterpart. Other examples are HESS J1303-631 (Aharonian et al. 2005c), the second unidentified $\mathrm{TeV}$ source, and the unidentified sources found in the HESS Galactic plane survey (Aharonian et al. 2005a). The identification of any of these sources, by locating its counterpart at radio, optical, or infrared wavelengths, would improve our knowledge of the emission processes at very high energies and would help to solve the problem of the unidentified $\mathrm{TeV}$ sources. TeV J2032+4130 is extended, with a radius of $6.2^{\prime}$, being the center of gravity (COG) of $\mathrm{TeV}$ photons located at $\alpha=20^{\mathrm{h}} 31^{\mathrm{m}} 57.0^{\mathrm{s}}$ and $\delta=+41^{\circ} 29^{\prime} 56.8^{\prime \prime}(\mathrm{J} 2000.0)$ with arcminute accuracy. Its emission exhibits a hard spectrum with a steady flux of about a few $10^{-12}$ ergs $\mathrm{cm}^{-2} \mathrm{~s}^{-1}$ (Aharonian et al. 2005d). The large angular size of the source, its steady flux, and the location in the Galactic plane point strongly to a Galactic nature. The absence of any evident counterpart led Aharonian et al. (2002) to propose as possible origins of the

\footnotetext{
${ }^{1}$ Departament d'Astronomia i Meteorologia, Facultat de Física, Universitat de Barcelona, Martí i Franquès, Barcelona, Spain; jmparedes@ub.edu.

${ }^{2}$ Departamento de Física, Escuela Politécnica Superior, Universidad de Jaén, Las Lagunillas, Jaén, Spain; jmarti@ujaen.es.

${ }^{3}$ National Center for Radio Astrophysics, TIFR, Ganeshkhind, Pune, India; ishwar@ncra.tifr.res.in.

${ }^{4}$ Max-Planck-Institut für Kernphysik, Heidelberg, Germany; vbosch@mpihd.mpg.de.
}

$\mathrm{TeV}$ emission the star association Cygnus OB2 or a jet-driven termination shock. Hadronic interactions within the innermost region of the winds of $\mathrm{O}$ and $\mathrm{B}$ stars can also produce significant gamma-ray luminosities at $\mathrm{TeV}$ energies, compatible with those found in TeV J2032+4130 (Torres et al. 2004; Domingo-Santamaría \& Torres 2006).

Despite the intensive observational campaigns undertaken for this source at different wavelengths, no obvious counterpart at radio, optical, or X-ray energies has been reported up to now, leaving TeV J2032+4130 presently unidentified (Butt et al. 2003, 2006; Mukherjee et al. 2003). Here we report the discovery of a population of radio sources well within the $1 \sigma$ radius of the TeV J2032+4130 extended emission, presenting some of these radio sources' optical/IR and X-ray counterparts. One or several of these objects, including both an extended diffuse component and compact radio sources, could be related to the very high energy emission. Their observational properties and nature are presented and discussed in $\S \S 2$ and 3.

\section{OBSERVATIONS AND ANALYSIS}

\subsection{Radio}

Most of the data presented in this work were obtained through observations performed with the Giant Metrewave Radio Telescope (GMRT) at the National Centre for Radio Astrophysics (NCRA) in Khodad, India, during 2005 July 9, 2005 September 1 , and 2006 July 18. We observed at the $610 \mathrm{MHz}$ frequency (49 $\mathrm{cm}$ wavelength) in spectral line mode with 128 channels covering a $16 \mathrm{MHz}$ bandwidth. The calibration of amplitude and bandpass was achieved by observing 3C 286 and 3C 48, while phase calibration was performed through repeated scans on the nearby phase calibrator J2052+365. Correction of the GMRT flux densities for the increase in the sky temperature in the TeV $\mathrm{J} 2032+4130$ direction was also taken into account. In addition, we also used archive data obtained during 2003 April 29 using the Very Large Array (VLA) of the National Radio Astronomy Observatory (NRAO) in New Mexico. The VLA was in its most compact $\mathrm{D}$ configuration, and the observations were carried out at 1.465 and $4.885 \mathrm{GHz}$ (20 and $6 \mathrm{~cm}$ wavelength, respectively). The VLA amplitude calibrator was 3C 48; J2052+365 and J2007+404 were used as phase calibrators. Both the GMRT and VLA data were processed using standard procedures within the AIPS software package of NRAO.

The results of the radio observations are presented in Fig- 


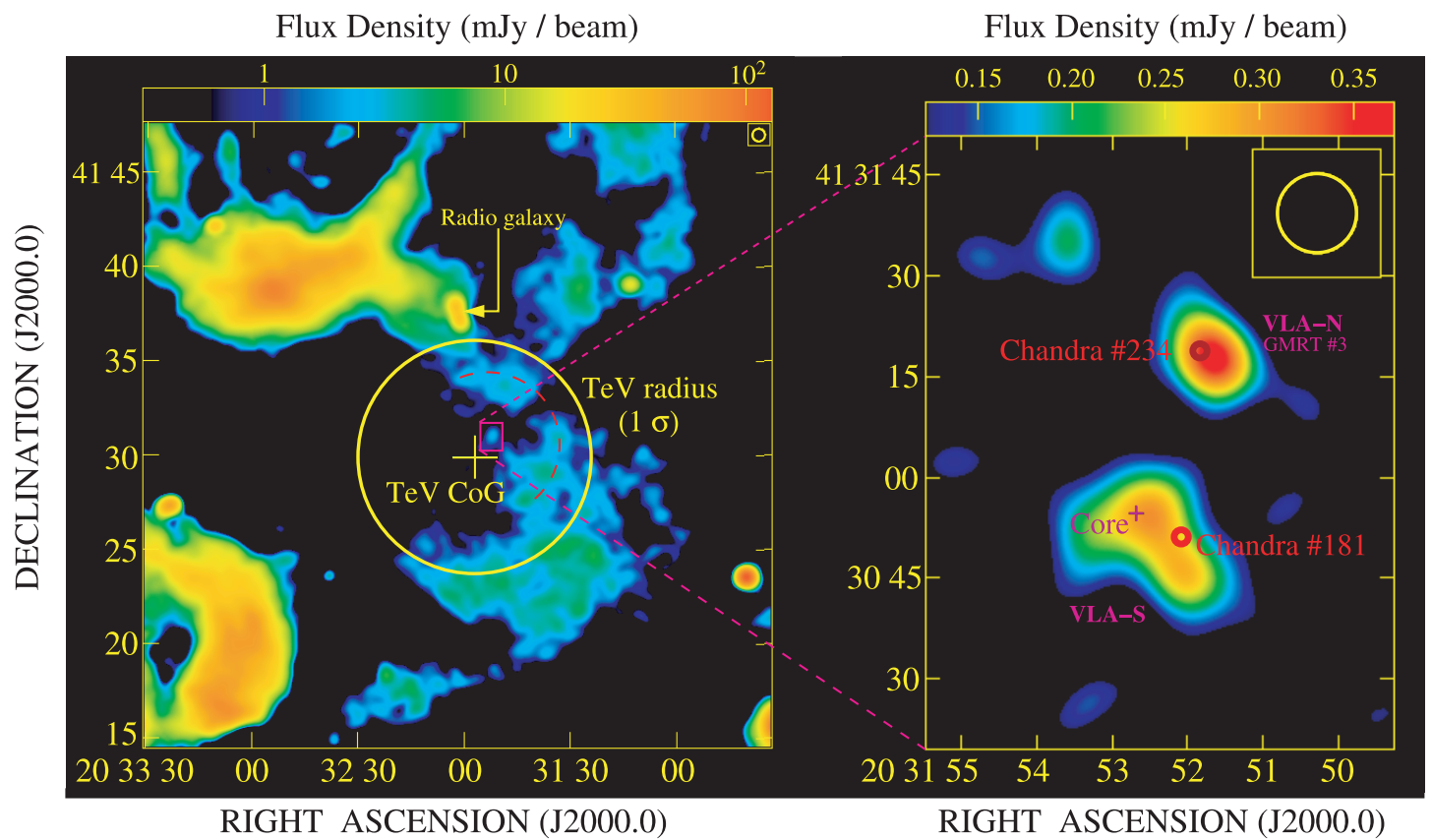

FIG. 1.-Left: Large-scale VLA mosaic, at the $20 \mathrm{~cm}$ wavelength $(1.4 \mathrm{GHz})$ in D configuration and uniform weight, of the unidentified gamma-ray source TeV $\mathrm{J} 2032+4130$. The COG of TeV emission and its statistical error are indicated by the central cross, while the big yellow circle illustrates the $1 \sigma$ radius of the $\mathrm{TeV}$ extended emission. Inside it, diffuse radio emission is clearly seen. The red half-arc traces the extended emission around the TeV COG position. Color scale goes logarithmically from above 0 to $126 \mathrm{mJy}$. The synthesized beam is shown at the top right corner as an $40.7^{\prime \prime} \times 40.2^{\prime \prime}$ ellipse, with position angle of $15.3^{\circ}$. Right: Zoomed map of an interesting area inside the TeV extended emission circle where a complex of VLA radio sources has been detected at the $6 \mathrm{~cm}$ wavelength $(4.8 \mathrm{GHz})$. Here the red circles indicate the location of Chandra X-ray sources in the field, labeled according to the X-ray identification number given by Butt et al. (2006). Chandra source 234, at $\alpha=20^{\mathrm{h}} 31^{\mathrm{m}} 51.84^{\mathrm{s}}$ and $\delta=+41^{\circ} 31^{\prime} 18.84^{\prime \prime}(\mathrm{J} 2000.0)$, is a hard X-ray source and is one of the strongest sources in the field of $\mathrm{TeV} 2032+4130$. This source has also been detected at $610 \mathrm{MHz}$ (source 3 in Table 1). Chandra source 181 , at $\alpha=20^{\mathrm{h}} 31^{\mathrm{m}} 52.08^{\mathrm{s}}$ and $\delta=+41^{\circ} 30^{\prime} 51.12^{\prime \prime}$ (J2000.0), is a weak source. The small cross shows the location, at $\alpha=20^{\mathrm{h}} 31^{\mathrm{m}} 52.686^{\mathrm{s}}$ and $\delta=+41^{\circ} 30^{\prime} 54.56^{\prime \prime}(\mathrm{J} 2000.0)$, of a compact radio core detected at $3.5 \mathrm{~cm}$ during a follow-up run with the VLA in A configuration (not shown here). Uniform weight was used to create this $6 \mathrm{~cm}$ map with a synthesized beam of $11.9^{\prime \prime} \times 11.8^{\prime \prime}$, with position angle of $-13.0^{\circ}$. The color scale goes linearly from nearly zero to $0.37 \mathrm{mJy} \mathrm{beam}^{-1}$.

ures 1 and 2. The left panel of Figure 1 contains a wide field map of the TeV $\mathbf{J} 2032+4130$ region obtained by mosaicking four individual pointings with the VLA at $20 \mathrm{~cm}$. Extended and diffuse radio emission is clearly seen inside the $\mathrm{TeV}$ $\mathrm{J} 2032+41301 \sigma$ radius and closely surrounding the COG of $\mathrm{TeV}$ photons, indicated by the central cross. The overall mor- phology of the main extended emission features in the field is also detected in GMRT maps (not shown here) retaining short spacings, confirming their shape and extension. A spectral index image (not shown here) created using matching beam VLA maps at 20 and $6 \mathrm{~cm}$ strongly suggests that there is a diffuse emission half-surrounding the COG of the TeV J2032+4130
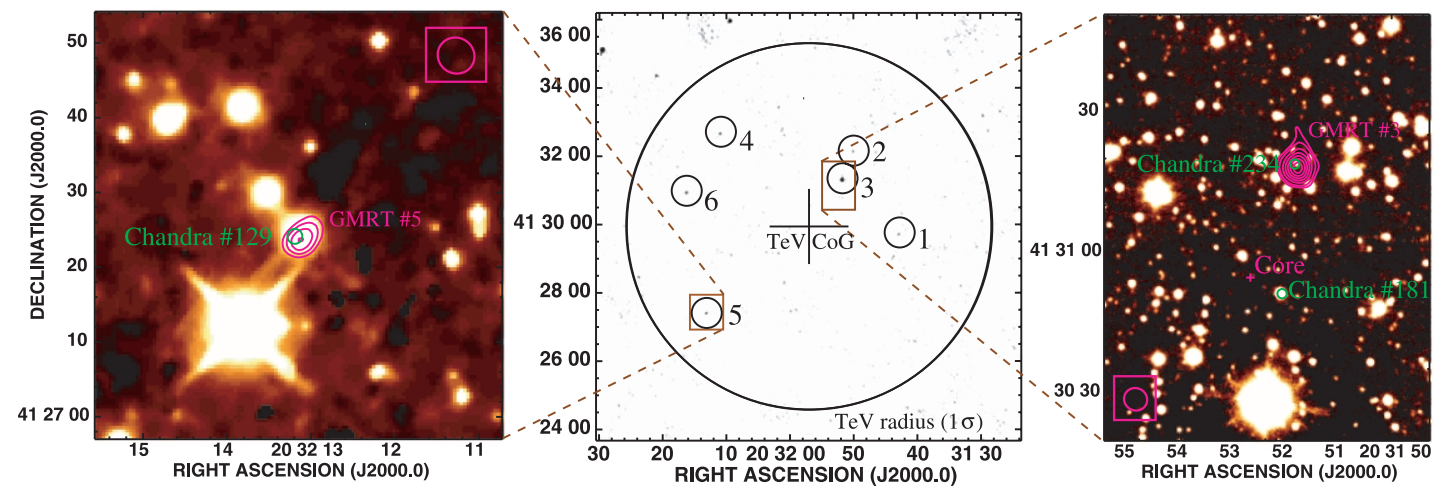

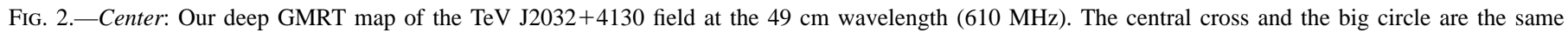

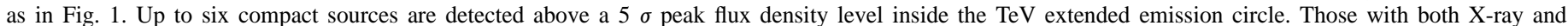

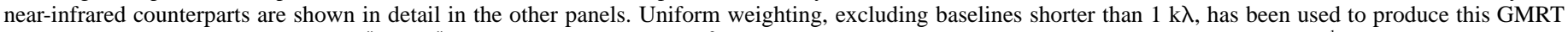

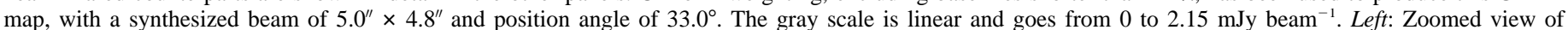

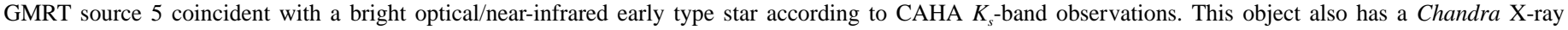

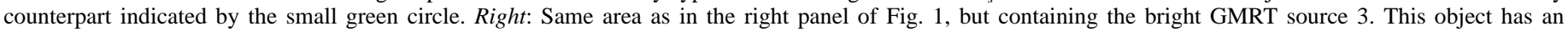

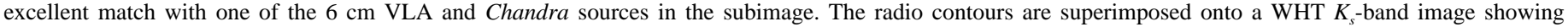
that there is also a faint near-infrared counterpart. 
TABLE 1

GMRT 610 MHz Radio Sources above $5 \sigma$ Peak Level

\begin{tabular}{lcccr}
\hline \hline Identification & $\begin{array}{c}\alpha \\
(\mathrm{J} 2000.0)\end{array}$ & $\begin{array}{c}\delta \\
(\mathrm{J} 2000.0)\end{array}$ & $\begin{array}{c}\text { Peak Flux Density } \\
(\mu \mathrm{Jy})\end{array}$ & $\begin{array}{c}\text { Integrated Flux Density } \\
(\mu \mathrm{Jy})\end{array}$ \\
\hline $1 \ldots \ldots \ldots \ldots$ & 203142.90 & +412942.7 & $478 \pm 83$ & $478 \pm 144$ \\
$2 \ldots \ldots \ldots \ldots$ & 203150.07 & +413208.4 & $454 \pm 85$ & $501 \pm 155$ \\
$3^{\mathrm{a}} \ldots \ldots \ldots \ldots$ & 203151.78 & +413118.3 & $1148 \pm 82$ & $1749 \pm 189$ \\
$4 \ldots \ldots \ldots \ldots$ & 203210.98 & +413239.6 & $521 \pm 85$ & $504 \pm 142$ \\
$5^{\mathrm{b}} \ldots \ldots \ldots \ldots$ & 203213.09 & +412724.5 & $525 \pm 82$ & $718 \pm 178$ \\
$6 \ldots \ldots \ldots \ldots$ & 203216.29 & +413055.6 & $566 \pm 82$ & $772 \pm 176$ \\
\hline
\end{tabular}

NoTE. - Units of right ascension are hours, minutes, and seconds, and units of declination are degrees, arcminutes, and arcseconds.

${ }^{\text {a }}$ Coincident with VLA-N, Chandra 234, and near-IR counterpart.

${ }^{\mathrm{b}}$ Coincident with Chandra 129 and stellar optical/near-IR counterpart.

source (Fig. 1, left, red dashed arc) with nonthermal spectral index $\alpha \simeq-1$, defined as $S_{\nu} \propto \nu^{\alpha}$. We note that the diffuse emission seen well outside the $\mathrm{TeV}$ source circle is thermal, hinting at a differentiated origin for the nonthermal diffuse radiation within the $\mathrm{TeV}$ source circle and a physical link between the diffuse radio mission and the $\mathrm{TeV}$ source. Another remarkable object in the field is a prominent double-lobe radio galaxy whose core is approximately at $\alpha=20^{\mathrm{h}} 32^{\mathrm{m}} 01.7^{\mathrm{s}}$ and $\delta=+41^{\circ} 37^{\prime} 22^{\prime \prime}(\mathrm{J} 2000.0)$, with an integrated flux density of $91 \pm 1 \mathrm{mJy}$ at the $20 \mathrm{~cm}$ wavelength.

The right panel of Figure 1 illustrates a zoomed VLA map at $6 \mathrm{~cm}$ of a region close to the TeV COG. At this shorter wavelength we can clearly detect here two radio sources with different morphology. The northern one (VLA-N) is practically compact, while the southern VLA source (VLA-S) is not consistent with being pointlike.

The most compact source sensitive radio view of the $\mathrm{TeV}$ $\mathrm{J} 2032+4130$ field comes nevertheless from the final $610 \mathrm{MHz}$ map combining all the different GMRT observing epochs. This map is presented as a gray-scale plot in the central panel of Figure 2 and provides the deepest radio image with arcsecond detail ever obtained thus far of the TeV J2032+4130 position. Extended emission has been removed by not including visibilities corresponding to baselines shorter than $1 \mathrm{k} \lambda=490 \mathrm{~m}$. The field shown covers the entire $1 \sigma$ radius, and at least six compact radio sources are detected with peak flux density above 5 times the rms noise of about $90 \mu \mathrm{Jy}$ beam $^{-1}$. Their observed properties are listed in Table 1.

Finally, we also mention that a short VLA exploratory time proposal in A configuration was also conducted by us at the $3.5 \mathrm{~cm}$ wavelength on 2006 April 1 and 3. As a result, a compact (angular size $\leq 0.3^{\prime \prime}$ ) radio source was detected with a flux density of $0.24 \pm 0.03 \mathrm{mJy}$ located at $\alpha=$ $20^{\mathrm{h}} 31^{\mathrm{m}} 52.686^{\mathrm{s}}$ and $\delta=+41^{\circ} 30^{\prime} 54.56^{\prime \prime}$ (J2000.0). This corresponds to the small cross in the right panel of Figure 1 (within the radio contours of the mentioned VLA-S source), with a "Core" label for reasons discussed below.

\subsection{Infrared}

The field of $\mathrm{TeV} \mathrm{J} 2032+4130$ was observed in the nearinfrared $K_{s}$ band $(2.2 \mu \mathrm{m})$ using the Calar Alto $3.5 \mathrm{~m}$ telescope and the OMEGA2000 camera on 2005 April 29. Another $K_{s^{-}}$ band image of the field was taken on 2006 July 7 using the $4.2 \mathrm{~m}$ William Herschel Telescope (WHT) and the LIRIS imager/spectrograph. These observations were processed in a standard way as described in Martí et al. (2006) with the IRAF package of the National Optical Astronomy Observatories (NOAO), including sky background subtraction and flat-fielding. The left and right panels of Figure 2 display the part of these observations showing the identification of near-infrared counterparts to some of the radio sources reported in this Letter. The identification is based on astrometric coincidence within a few tenths of an arcsecond.

\section{RESULTS AND DISCUSSION}

An important consideration concerning the new radio sources reported here is their coincidence with X-ray emitters in the field previously reported by other authors (Butt et al. 2003, 2006; Mukherjee et al. 2003). Hereafter, X-ray sources will be referred to using the Chandra Butt et al. (2006) identification numbers. We discuss this issue for the three most relevant objects reported in this work, namely, the VLA-N source (or GMRT source 3), the VLA-S source (undetected by the GMRT), and the GMRT source 5. VLA-N and VLA-S are well detected separately only at $6 \mathrm{~cm}$, but an estimate of their individual $20 \mathrm{~cm}$ flux densities is feasible by fitting two Gaussian components with fixed positions.

VLA-N is well within the $1^{\prime \prime}$ uncertainty of Chandra $234 \mathrm{X}$ ray source, and it also has a faint $K_{s}=16.8 \pm 0.1$ counterpart in our WHT image (see the right panels of Figs. 1 and 2). Combining VLA+GMRT data, the radio spectrum of this northern VLA source can be described by $S_{v}=(1.35 \pm 0.06) \mathrm{mJy}$ $(\nu / \mathrm{GHz})^{-0.47 \pm 0.03}$ and is therefore clearly a nonthermal emitter. The fit to the Chandra 234 spectrum gives a hard photon index of $1.1 \pm 0.4$, an unabsorbed flux of $\sim 2 \times 10^{-13} \mathrm{ergs} \mathrm{cm}^{-2} \mathrm{~s}^{-1}$ in the range $2-10 \mathrm{keV}$, and a hydrogen column density of $\sim 2 \times$ $10^{22} \mathrm{~cm}^{-2}$. We note that the data are poor and do not allow for detailed fitting. Assuming the accepted distance to the Cygnus OB2 association of $1.7 \mathrm{kpc}$, the X-ray luminosity in the range 2$10 \mathrm{keV}$ would be $\sim 10^{32} \mathrm{ergs} \mathrm{s}^{-1}$. Similarly, the $0.1-100 \mathrm{GHz}$ radio luminosity is estimated as $L_{\mathrm{rad}}=1.0 \times 10^{29} \mathrm{ergs} \mathrm{s}^{-1}$. These values have to be considered as lower limits if the source is beyond the Cygnus OB2 distance.

VLA-S is also of nonthermal nature, with its radio spectrum given by $S_{\nu}=(2.6 \pm 0.6) \mathrm{mJy}(\nu / \mathrm{GHz})^{-0.5 \pm 0.2}$ from VLA data only. Undetected by the GMRT above $0.27 \mathrm{mJy}(3 \sigma)$, its spectral index must be significantly inverted, i.e., with $\alpha \geq+1.0$ between 49 and $20 \mathrm{~cm}$. This fact is suggestive of synchrotron emission from an optically thick core at long wavelengths. Moreover, this object also appears superposed onto the Chandra 181 error circle (see again the right panels of Figs. 1 and 2). Since the number of X-ray counts is too low to perform a proper spectral analysis, we assume a photon index of 1.5 , a hydrogen column density of $\sim 10^{22} \mathrm{~cm}^{-2}$, and the same distance as above, deriving an Xray luminosity in the range $2-10 \mathrm{keV}$ of about $10^{31} \mathrm{ergs} \mathrm{s}^{-1}$. For the optically thin radio luminosity, we obtain $L_{\text {rad }}=1.6 \times$ $10^{29} \mathrm{ergs} \mathrm{s}^{-1}$. Inside the Chandra 181 circle, there is a $K_{s}=$ $13.9 \pm 0.1$ near-infrared source that is likely of stellar nature, 
based on WHT spectroscopy showing no lines with cosmological redshift. We propose it to be the counterpart of the X-ray source, provided that the marginal Chandra 181 detection is real. However, the complex morphology of the VLA-S radio source (see Fig. 1, right) suggests that it consists of at least two different components barely resolved in the D configuration of the array. One of them could be the Chandra X-ray/IR source and the other the compact radio source detected in our VLA exploratory observation in A configuration at $3.5 \mathrm{~cm}$. The other one is possibly related to the compact core just mentioned above, but further confirmation is yet necessary. The Galactic or extragalactic nature of the mentioned radio core is impossible to establish from infrared spectroscopy due its extreme faintness in our WHT image $\left(K_{s}=19.2 \pm 0.3\right)$.

Another remarkable coincidence is that of the GMRT source 5, whose position agrees very well with a $V=11.95$ and $K_{s}=9.07 \pm 0.02$ optical/infrared star belonging to the Cygnus OB2 association. This object is catalogued as star MT91 213 with a B0 Vp spectral type (Massey \& Thompson 1991; Butt et al. 2003). There is also the Chandra 129 source at the position of such a bright object (see error circle in the left panel of Fig. 2). With the same assumptions as above, this star has an X-ray luminosity of about $3 \times 10^{31} \mathrm{ergs} \mathrm{s}^{-1}$ in the same energy range. From its non-VLA detection at $6 \mathrm{~cm}$ and excluding variability, we obtain a spectral index constraint of $\alpha \leq-0.76$ that points again to a nonthermal emission mechanism with a radio luminosity of $L_{\text {rad }}(0.1-100 \mathrm{GHz})=1.7 \times 10^{28} \mathrm{ergs} \mathrm{s}^{-1}$. Colliding winds in an early-type binary or gyrosynchrotron radiation from mildly relativistic electrons trapped in the strong stellar magnetic field could be responsible for the observed nonthermal emission in this peculiar spectrum star.

We recall that all the luminosities provided here are just lower limits if the sources are farther than Cygnus OB2. In fact, only GMRT source 5 is a known member of the association, but the others could be more distant. For instance, at $10 \mathrm{kpc}$ these objects would present luminosities almost 2 orders of magnitude larger, likely requiring the presence of a compact object, and thus pointing to an X-ray binary nature (for a model of microquasars$\mathrm{X}$-ray binaries with jets-powering extended TeV hadronic emission, see Bosch-Ramon et al. [2005]).

At this point, we should not forget the existence of the extended source present in the $20 \mathrm{~cm}$ VLA map around the TeV COG. This feature covers a solid angle of about $27 \mathrm{arcmin}^{2}$ and its radius would correspond to $\sim 2 \mathrm{pc}$ at the Cygnus OB2 distance. An integrated flux density of $116 \pm 3 \mathrm{mJy}$ is obtained at $20 \mathrm{~cm}$. For synchrotron emission with $\alpha=-1$ at the Cygnus OB2 distance, its total radio luminosity between 0.1 and $100 \mathrm{GHz}$ amounts to $L_{\mathrm{rad}} \simeq 3.9 \times 10^{30} \mathrm{ergs} \mathrm{s}^{-1}$. From equipartition consideration, one can also derive a total energy content of $\sim 6.4 \times 10^{45} \mathrm{ergs}$ and a magnetic field of $1.6 \times 10^{-5} \mathrm{G}$. The energetics would point to an efficient injector of nonthermal particles, which is consistent with the fact that the region emits at $\mathrm{TeV}$. The magnetic field value is consistent with a slight en- hancement of the interstellar medium (ISM) density value. All this could be explained by a shock driven by a weakly radiative jet in the ISM produced by some of the pointlike sources, perhaps possible microquasars, within the $\mathrm{TeV}$ region. In any case, the radio flux densities reported here provide a useful upper limit to the low-energy counterpart of the $\mathrm{TeV}$ source (see also $\mathrm{Mu}$ kherjee et al. [2006] for a diffuse X-ray component inside the $\mathrm{TeV}$ source region presenting fluxes of $\sim 10^{-13} \mathrm{ergs} \mathrm{cm}^{-2} \mathrm{~s}^{-1}$ ) that allow, and call for, further constraining the models put forward so far.

To summarize, our results reveal that at least three nonthermal radio sources, one of them being a significantly hard X-ray source, and a nonthermal diffuse radio component are detected in the line of sight toward TeV J2032+4130. These facts completely change the situation about this unidentified extended $\mathrm{TeV}$ source. Where no counterpart was seen before, now several radio sources have emerged and future observations should decide whether or not one of them is related to the $\mathrm{TeV}$ emission. All radio/X-ray sources have significantly negative spectral indices, suggestive of optically thin synchrotron emission. Two of these sources are also likely of stellar origin, and one is certainly a bright early-type star in Cygnus OB2. Excluding this last case, the distance to the rest is not yet clearly established. Thus, their $\mathrm{X}$-ray luminosities may be comparable to those of X-ray binaries if placed beyond Cygnus OB2.

Are one or more of these objects similar to the gamma-ray binaries LS 5039 (Paredes et al. 2000; Aharonian et al. 2005b) and LS I+61303 (Albert et al. 2006)? With a farther location in the Galaxy, our sources would have similar radio and X-ray luminosities and, if powering the $\mathrm{TeV}$ extended radiation via, for example, shock with the ISM, the energetic reservoir would be similar as well (see, e.g., Paredes et al. 2006). An in-depth exploration of all these scenarios is beyond the scope of this Letter and will be addressed in a future work.

The authors acknowledge support by DGI of the Ministerio de Educación y Ciencia, Spain, under grants AYA2004-07171C02-01 and AYA2004-07171-C02-02 and FEDER funds. J. M. is also supported by Plan Andaluz de Investigación of Junta de Andalucía as research group FQM322. GMRT is run by the National Centre for Radio Astrophysics of the Tata Institute of Fundamental Research. The National Radio Astronomy Observatory is a facility of the National Science Foundation operated under cooperative agreement by Associated Universities, Inc. This Letter is also based on observations collected at the Centro Astronómico Hispano Alemán (CAHA) at Calar Alto, operated jointly by the Max-Planck-Institut für Astronomie and the Instituto de Astrofísica de Andalucía (CSIC). The William Herschel Telescope is operated on the island of La Palma by the Isaac Newton Group in the Spanish Observatorio del Roque de los Muchachos of the Instituto de Astrofísica de Canarias.

Facilities: GMRT, VLA, ING:Herschel, CAO:3.5m

\section{REFERENCES}

Aharonian, F., et al. 2002, A\&A, 393, L37

2005a, Science, 307, 1938

2005b, Science, 309, 746

2005c, A\&A, 439, 1013

2005d, A\&A, 431, 197

2006, ApJ, 636, 777

Albert, J., et al. 2006, Science, 312, 1771

Bosch-Ramon, V., Aharonian, F., \& Paredes, J. M. 2005, A\&A, 432, 609

Butt, Y., Drake, J., Benaglia, P., Combi, J. A., Dame, T., Miniati, F., \& Romero,

G. E. 2006, ApJ, 643, 238

Butt, Y., et al. 2003, ApJ, 597, 494
Domingo-Santamaría, E., \& Torres, D. F. 2006, A\&A, 448, 613

Martí, J., Pérez-Ramírez, D., Luque-Escamilla, P., Garrido, J. L., Paredes, J. M., Muñoz-Arjonilla, A. J., \& Sánchez-Sutil, J. R. 2006, A\&A, 451, 1037 Massey, P., \& Thompson, A. B. 1991, AJ, 101, 1408

Mukherjee, R., Halpern, J. P., \& Gotthelf, E. V. 2006, Ap\&SS, in press (astro$\mathrm{ph} / 0610299)$

Mukherjee, R., Halpern, J. P., Gotthelf, E. V., Eracleous, M., \& Mirabal, N. 2003, ApJ, 589, 487

Paredes, J. M., Bosch-Ramon, V., Romero, G. E, 2006, A\&A, 451, 259

Paredes, J. M., Martí, J., Ribó, M., \& Massi, M. 2000, Science, 288, 2340

Torres, D. F., Domingo-Santamaría, E., \& Romero, G. E. 2004, ApJ, 601, L75 